\title{
Tendencies for higher co-expression of EGFR and HER2 and downregulation of HER3 in prostate cancer lymph node metastases compared with corresponding primary tumors
}

\author{
J.CARLSSON ${ }^{1}$, L. SHEN $^{2}$, J. XIANG ${ }^{2}$, J. XU ${ }^{2}$ and Q. $\mathrm{WEI}^{2}$ \\ ${ }^{1}$ Unit of Biomedical Radiation Science, Department of Radiology, Oncology and Radiation Science, \\ Rudbeck Laboratory, Uppsala University, SE-751 85 Uppsala, Sweden; \\ ${ }^{2}$ Department of Radiation Oncology, The Second Affiliated Hospital and Cancer Institute \\ (National Ministry of Education Key Laboratory of Cancer Prevention and Intervention), \\ Zhejiang University School of Medicine, Hangzhou 310009, P.R. China
}

Received May 8, 2012; Accepted August 8, 2012

DOI: $10.3892 / \mathrm{ol} .2012 .996$

\begin{abstract}
The epidermal growth factor receptor (EGFR) family members are potential targets for therapy using extracellular domain receptor binding agents, such as the antibodies trastuzumab and cetuximab, or antibodies labeled with therapeutically useful radionuclides or toxins. This is especially the case when the tumor cells are resistant to chemotherapy and tyrosine kinase inhibitors. Studies concerning the expression of these receptors in prostate cancer vary in the literature, possibly due to differences in patient inclusion, sample preparations and scoring criteria. In our study, EGFR, HER2 and HER3 expression was analyzed in prostate cancer samples from primary tumors and corresponding lymph node metastases from 12 patients. The expression of HER2 and EGFR was scored from immunohistochemical preparations and the HercepTest criteria $(0,1+, 2+$ or $3+)$, while HER3 expression was scored as no, weak or strong staining. There were 5 EGFR-positive (2+ or $3+)$ primary tumors and 6 EGFR-positive lymph node metastases, and there was EGFR upregulation in one metastasis. Only 4 of the 12 patients had marked HER 2 expression $(2+$ or $3+)$ in their primary tumors and there was one downregulation and 5 cases of upregulation in the metastases. Thus, a total of 8 out of 12 analyzed metastases were HER2-positive. Of the 12 primary tumors, 9 expressed HER 3 while only 2 of the lymph node metastases expressed recognizable HER3 staining, so 7 metastases appeared to have downregulated HER3 expression.
\end{abstract}

Correspondence to: Professor Jörgen Carlsson, Unit of Biomedical Radiation Science, Department of Radiology, Oncology and Radiation Science, Rudbeck Laboratory, Uppsala University, Dag Hammarskjolds vag 20, SE-751 85 Uppsala, Sweden

E-mail: jorgen.carlsson@bms.uu.se

Key words: cancer, EGFR, HER2, HER3, lymph nodes, metastasis, prostate, radionuclides, receptor expression, therapy
In one of the primary tumors there was positive co-expression of EGFR and HER2, while this co-expression was observed in 4 of the metastases. Thus, there were tendencies for upregulation of HER2, increased co-expression of EGFR and HER2 and downregulation of HER3 in the prostate cancer lymph node metastases in comparison to the primary tumors. The results are encouraging for studies involving more patients. Possible strategies for EGFR- and HER2-targeted therapy are briefly discussed in the present study, especially with regard to the expression and co-expression of EGFR and HER2 in metastases.

\section{Introduction}

A number of prostate cancer patients have metastatic growth at diagnosis and others develop metastases after potentially curative surgery or radiotherapy. Combinations of chemotherapy agents have some efficacy in these cases, but the prognosis for long-term survival is poor, especially when the tumors have formed distant metastases, e.g., in the skeleton. Receptor-targeted therapy with radionuclides or toxins may improve the response and survival times, especially in cases where chemotherapy and therapy with tyrosine kinase inhibitors are not effective. Targeted radionuclide therapy, supported by imaging for treatment planning, dosimetry and follow-up of therapy effects, is one option $(1,2)$.

In order for receptor-targeted therapy to be an effective complement or alternative to chemotherapy, the disseminated tumor cells and metastases must express the target structure to at least a similar extent as the primary tumors. There are several indications for various types of tumors that in cases where the expression of members of the epidermal growth factor receptor (EGFR) family is high in the primary tumor, it may also be high in the metastases (2-4). The reason for this may be that the receptor-expressing tumor cells require the growth factor-receptor interactions for growth stimulation. If disseminated tumor cells reduce or lose the expression of the 
receptor, for example due to genomic instability, they may also lose growth capacity $(3,5)$.

The EGFR family consists of EGFR, HER2, HER3 and HER4, which have an extracellular ligand binding domain, a hydrophobic transmembrane domain and an intracellular domain with protein-tyrosine kinase activity. However, HER3 has no intrinsic tyrosine kinase activity and no ligand for HER2 has been identified to date, but they both contribute to intracellular signaling via dimerization with each other or with other receptors in the family. EGF and five other ligands bind to EGFR and neuregulins (NRGs) are the ligands for HER3 and HER4. The overexpression of EGFR and HER2 has been reported to be associated with high malignancy (2-7).

Targeted therapy is a clinical reality for tumors which express EGFR (cetuximab) or HER2 (trastuzumab), although resistance has been reported in both cases (8-12). EGFR and HER2 appear to be good targets for radionuclide- or toxin-based tumor therapy, although whether this is the case for prostate cancer is not clear $(2,3)$. It remains to be determined whether HER3 is also a suitable target in prostate cancer (13). One problem appears to be that in immunohistochemical staining for several tumor types, including laryngeal, esophageal, base of tongue carcinomas and colorectal tumors, HER3 is often observed to be mainly localized to the cytoplasm (14-17) (see also the protein atlas: http://www.proteinatlas.org/). This staining pattern is not understood since HER3 contains a transmembrane region. The role of HER4 in tumor growth is not clear $(2,3)$ and therefore, HER4 was not analyzed in this study.

EGFR family-targeted radionuclide or toxin therapy aims to target the often abundant native, not mutated, receptors and the effect of such therapy is probably not dependent on whether the targeting agent strongly interferes with intracellular signaling. The cell killing properties of ionizing radiation and toxins are well known and treatment-induced resistance for radiation has, to the best of our knowledge, not been reported (2). With this background as inspiration, we investigated the expression of EGFR, HER2 and HER3 in 12 prostate cancer patients in primary tumors and corresponding lymph node metastases.

\section{Materials and methods}

Patients and samples. A total of 12 patients with lymph node-positive prostate cancer were diagnosed and treated in the Affiliated Hospitals, Zhejiang University School of Medicine (Hangzhou, China). They were included in this study after approval of the Institutional Review Board. Primary tumor and lymph node metastases samples were obtained from all patients following their consent.

The primary tumor tissues and the lymph node metastases samples were fixed in $4 \%$ buffered formalin, processed and embedded in paraffin. Sections, $4-\mu \mathrm{m}$ thick, were cut and deparaffinized in xylene and hydrated through graded concentrations of ethanol to distilled water (14-17). The samples were then stained with hematoxylin and eosin for routine clinical analysis and, for this specific research project, with immunohistochemical receptor staining, as described below. The patient and tumor characteristics are shown in Table I.

EGFR staining. EGFR was assessed by immunohistochemistry using a streptavidin-biotin complex technique as
Table I. Patient data.

\begin{tabular}{cccc}
\hline Patient & Age (years) & Gleason score & T-stage \\
\hline 1 & 63 & No record & T3 \\
2 & 67 & $4+4=8$ & T3 \\
3 & 58 & $4+5=9$ & T4 \\
4 & 69 & $4+4=8$ & T2 \\
5 & 64 & $3+5=8$ & T3 \\
6 & 63 & $5+4=9$ & T4 \\
7 & 66 & $4+5=9$ & T3 \\
8 & 68 & $3+4=7$ & T2 \\
9 & 74 & $5+3=8$ & T3 \\
10 & 59 & $4+5=9$ & T3 \\
11 & 61 & $3+4=7$ & T3 \\
12 & 57 & $5+4=9$ & T2
\end{tabular}

All patients had local lymph node metastases (N-stage, N1) but no recognizable distant metastases (M-stage, M0) at diagnosis. The Gleason scores were calculated according to Gleason $(18,19)$. The T-staging (20) was: T2, tumor is palpated and intact capsule was observed; T3, tumor has spread through the prostate capsule; T4, tumor has invaded nearby structures. All patients had local lymph node metastases, but few were observed to have intact capsule (T2 stage). This indicates direct spread via lymph vessel drainage without breaking the capsule.

previously described $(14,15,17)$. After deparaffinization of the sections, endogenous peroxidase was blocked in $0.3 \%$ $\mathrm{H}_{2} \mathrm{O}_{2}$ in PBS for $20 \mathrm{~min}$. Then, enzymatic antigen retrieval was performed in $0.05 \%$ protease K (Code no. S3020, Dako, Glostrup, Denmark) in PBS for $10 \mathrm{~min}$ at room temperature. The slides were preincubated in PBS for $10 \mathrm{~min}$. The primary mouse monoclonal antibody directed against EGFR (clone 31G7, Zymed Laboratories, South San Francisco, CA, USA) was diluted 1:100 and incubated overnight at $4^{\circ} \mathrm{C}$. The secondary biotinylated antibodies (goat anti-mouse, Dako) and the peroxidase-labeled streptavidin-biotin complex (Dako) were diluted 1:200 and incubated for $30 \mathrm{~min}$ at room temperature. All slides were developed in $0.05 \%$ diaminobenzidine (Sigma, St. Louis, MO, USA) for $5 \mathrm{~min}$ and counterstained in Harris hematoxylin (Sigma). Finally, the slides were dehydrated through graded alcohol to xylene and mounted in organic mounting medium (Pertex ${ }^{\circledR}$, Histolab, Gothenburg, Sweden).

HER2 staining. The HER2 immunohistochemical staining was performed as previously described $(14,15,17)$. After deparaffinization, the sections were incubated in methanol and hydrogen peroxide for $30 \mathrm{~min}$ to quench endogenous peroxidase activity. Antigen retrieval was performed in a waterbath at $95-98^{\circ} \mathrm{C}, \mathrm{pH} 6.0$, for $40 \mathrm{~min}$. The glasses were then cooled to room temperature and then washed in distilled water. Immunohistochemical stainings were performed using the Elite ABC Kit (Vectastain, Vector Laboratories, Burlingame, CA, USA). Blocking serum was applied for $15 \mathrm{~min}$ and followed by incubation with rabbit anti-human c-erbB-2 oncoprotein (code No. A 0485, Dako) diluted 1:350. Sections were 
then incubated with the biotinylated secondary antibody and visualized using the peroxidase substrate 3-amino-9-ethylcarbazole (AEC; Sigma A-5754) as a chromogen. Finally, the sections were counterstained with Mayer's hematoxylin and mounted with Aquamount (BDH Ltd., Poole, UK).

HER3 staining. The HER3 staining was performed as previously described $(14,15,17)$. After deparaffinization, the sections were incubated in methanol and hydrogen peroxide for $30 \mathrm{~min}$ to quench endogenous peroxidase activity. Antigen retrieval was performed in a pressure chamber at $125^{\circ} \mathrm{C}, \mathrm{pH} 9.0$, for $4 \mathrm{~min}$. The glasses were then cooled at room temperature and washed in distilled water. Immunohistochemical stainings were performed using the Elite ABC Kit (Vectastain, Vector Laboratories). Blocking serum was applied for $15 \mathrm{~min}$ and followed by incubation with the monoclonal antibody MAB4021 (Chemicon, Temecula, CA, USA) diluted 1:800. Sections were then incubated with a biotinylated secondary antibody and visualized using AEC as a chromogen. Finally, the sections were counterstained with Mayer's hematoxylin and mounted with Aquamount (BDH Ltd.).

EGFR and HER2 scores. The HER2 expression was scored using the HercepTest scoring criteria. This is based on a scale where 0 corresponds to tumor cells that were completely negative, $1+$ corresponds to faint perceptible staining of the tumor cell membranes, 2+ corresponds to moderate staining of the entire tumor cell membranes and $3+$ is marked circumferential staining of the entire tumor cell membranes creating a fishnet pattern. The Canadian and the Dako HercepTest guidelines (21) that require $>10 \%$ of the tumor cells to be stained were applied. Cytoplasmic staining was considered to be non-specific and was not included in the scoring. As positive controls we used in-house positive control tissue sections as well as positive control sections supplied by Dako. As negative HER2 controls we used normal tissues, which are expected not to express HER2, such as connective tissue observed in the same sections as the tumor cells. In the metastases sections we used lymphocytes and the surrounding capsule of the lymph nodes as HER2-negative internal controls. The expression pattern of EGFR is similar to that of HER2 and EGFR expression was therefore evaluated using the same scoring criteria as for HER2. As EGFR-positive controls we used in-house positive control skin tissue sections. As negative controls we used connective tissue observed in the same sections as the tumor cells. In the metastases sections we used lymphocytes and the surrounding capsule of the lymph nodes as EGFR-negative internal controls.

HER3 evaluation. The HER3 staining was evaluated as negative, weak or strong staining $(14,15,17)$. Negative corresponded to tumor cells that were not at all stained, weak corresponded to faint staining of the tumor cytoplasm with or without stained granules and strong corresponded to intensive tumor granular cytoplasmic staining. As positive controls we used normal laryngeal epithelium (positive reference staining may also be found at www.proteinatlas.org). As negative controls we used tumor stroma of connective tissue character and non-tumor invaded areas of lymph nodes.
Table II. EGFR family (EGFR, HER2 and HER3) scores in primary tumors and in the corresponding lymph node metastases.

A, EGFR and HER2 scores

\begin{tabular}{lcccc}
\hline & \multicolumn{4}{c}{ Metastasis (patient number) } \\
\cline { 2 - 5 } Primary tumor & 0 & $1+$ & $2+$ & $3+$ \\
\hline EGFR & 3,6 & - & - & - \\
0 & $5,9,10,11$ & - & - & 7 \\
$1+$ & - & - & $1,8,12$ & - \\
$2+$ & - & - & - & 2,4 \\
$3+$ & & & & \\
HER2 & 1,11 & - & - & - \\
0 & 8 & - & $2,5,6,7$ & 4 \\
$1+$ & 9 & - & 3,10 & - \\
$2+$ & - & - & - & 12 \\
$3+$ & & & & \\
\hline
\end{tabular}

B, HER3 scores

\begin{tabular}{lccc}
\hline & \multicolumn{3}{c}{ Metastasis (patient number) } \\
\cline { 2 - 4 } Primary tumor & Negative & Weak & Strong \\
\hline Negative & $1,2,3$ & - & - \\
Weak & $5,6,8,9,10,12$ & - & 4 \\
Strong & 11 & 7 & - \\
\hline
\end{tabular}

Scoring criteria are described in Materials and methods. Patient numbers are as listed in Table I. EGFR, epidermal growth factor receptor.

\section{Results}

Since only 12 patients were included in the study (Table I), we report results for the primary tumors and metastases of each individual patient in Tables II-IV.

EGFR expression. Of the 12 primary tumors, 5 had $2+$ or $3+$ EGFR scores (patients 1, 2, 4, 8 and 12). The EGFR expression in the corresponding lymph node metastases was upregulated in one metastasis (patient 7), so a total of 6 metastases were EGFR-positive. No downregulation of EGFR from $2+$ or $3+$ in the primary tumor to 0 or $1+$ in the metastasis was observed (Table IIA).

HER 2 expression. Of the 12 patients, 4 had strong HER2 expression $(2+$ or $3+$ ) in the primary tumors (patients $3,9,10$ and 12). There was one downregulation (from $2+$ to 0 , patient 9) and 5 cases of upregulation in the metastases (patients 2, 4, 5, 6 and 7), giving a total of 8 HER2-positive metastases out of the 12 cases analyzed. Thus, there was a marked tendency for the upregulation of HER2 in the metastases (Table IIA).

HER3 expression. HER3 was weakly or strongly expressed in 9 out of the 12 cases in the primary tumors (all except patients 1,2 , and 3) but downregulated in all but two (patients 4 and 7) 
Table III. Co-expression of the receptors.

\begin{tabular}{lccc}
\hline & \multicolumn{2}{c}{ Patient number } & \\
\cline { 2 - 3 } Tissue & Positive & Negative & $\begin{array}{c}\text { No. of } \\
\text { co-expression }\end{array}$ \\
\hline Primary tumors & & & \\
EGFR, HER2, HER3 & 12 & - & 1 \\
EGFR, HER2 & 12 & $5,6,7,11$ & 5 \\
EGFR, HER3 & $4,8,12$ & 3 & 4 \\
HER2, HER3 & $9,10,12$ & 1,2 & 5 \\
Metastases & & & \\
EGFR, HER2, HER3 & 4,7 & 9,11 & 4 \\
EGFR, HER2 & $2,4,7,12$ & 9,11 & 6 \\
EGFR, HER3 & 4,7 & $3,5,6,9,10,11$ & 8 \\
HER2, HER3 & 4,7 & $1,8,9,11$ & 6
\end{tabular}

EGFR and HER2 were considered to be positive at $2+$ or $3+$. HER3 was considered to be positive when scored weak or strong (see Materials and methods). Patient numbers are as listed in Table I. EGFR, epidermal growth factor receptor.

Table IV. Correlation between EGFR and HER2 in the studied primary tumor and metastasis samples.

\begin{tabular}{lcccc}
\hline & \multicolumn{4}{c}{ HER2 (patient number) } \\
\cline { 2 - 5 } EGFR & 0 & $1+$ & $2+$ & $3+$ \\
\hline Primary tumor & - & 6 & 3 & - \\
0 & 11 & 5,7 & 9,10 & - \\
$1+$ & 1 & 8 & - & $\underline{12}$ \\
$2+$ & - & 2,4 & - & - \\
$3+$ & & & & \\
Metastasis & 9,11 & - & $3,5,6,10$ & - \\
0 & - & - & & - \\
$1+$ & 1,8 & - & - & $\underline{12}$ \\
$2+$ & - & - & $\underline{2,7}$ & $\underline{4}$ \\
$3+$ & & &
\end{tabular}

Patients with positive expression in both EGFR and HER2 are marked with italics and underlined. For the primary tumors this was the case for only patient 12 , while patients 2, 4, 7 and 12 had positive expression of both EGFR and HER2 in their analyzed metastases, indicating that only these are candidates for combined EGFR- and HER2-targeted therapy. Patient numbers are as listed in Table I. EGFR, epidermal growth factor receptor.

in the corresponding lymph node metastases. Thus, 7 metastases had downregulated HER3 compared with the primary tumors (Table IIB).

Co-expression. There was positive co-expression of all three receptors in only one of the primary tumors (patient 12) and that patient was also the only one with positive co-expression of EGFR and HER2 (2+ and 3+, respectively) when the

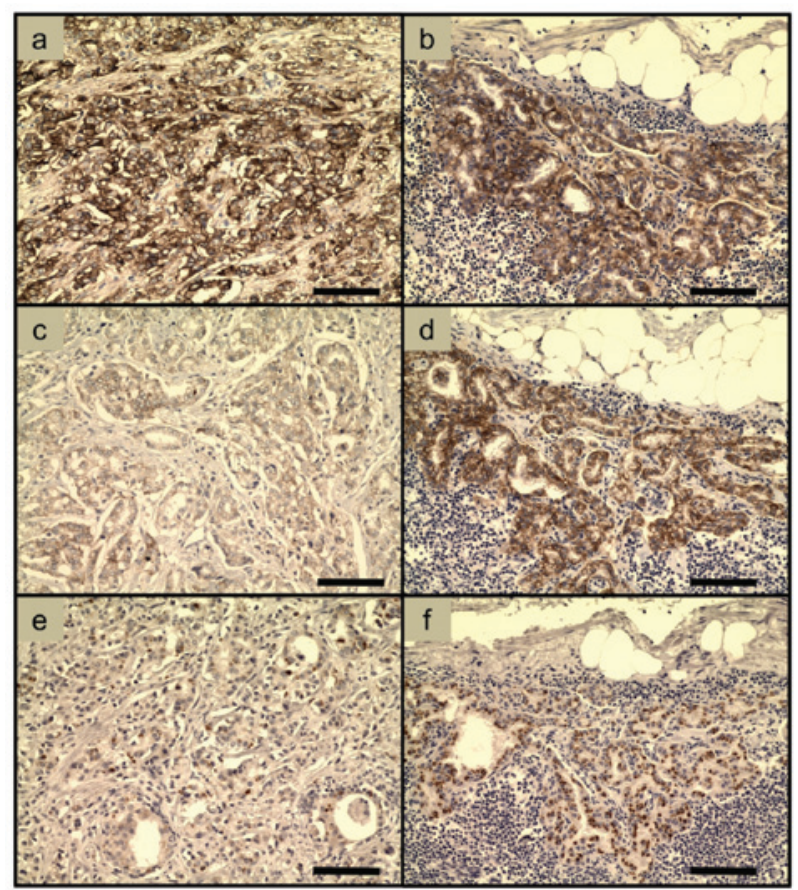

Figure 1. Examples of immunohistochemical stainings of samples from patient 4. (a) EGFR, primary tumor (3+). (b) EGFR, lymph node metastasis (3+). (c) HER2, primary tumor (1+). (d) HER2, lymph node metastasis (3+). (e) HER3, primary tumor (weak). (f) HER3, lymph node metastasis (strong). Note that the HER 3 staining in the samples from patient 4 is the only case in which HER3 appeared to be stronger in the metastasis than in the primary tumor. The scorings are as described in the text and in Table II. The bars correspond to $100 \mu \mathrm{m}$. EGFR, epidermal growth factor receptor.

primary tumors were considered. However, there was positive co-expression of EGFR and HER2 in 4 of the metastases (patients 2, 4, 7 and 12). Thus, there was a marked tendency that the positive co-expression of EGFR and HER2 increased in the lymph node metastases compared with the primary tumors. The extensive downregulation of HER3 in the metastases resulted in reduced co-expression of EGFR and HER3 (Tables III and IV).

Immunohistochemical stainings. Examples of immunohistochemical EGFR, HER2 and HER3 stainings of samples from patient 4 are shown in Fig. 1. Note the strong EGFR staining in both the primary tumor (Fig. 1a) and the metastasis (Fig. 1b). HER2 was upregulated in the metastasis (Fig. 1d) compared with the primary tumor (Fig. 1c). The HER3 staining changed from weak in the primary tumor (Fig. 1e) to strong in the metastasis (Fig. 1f), in contrast to the HER3 stainings in all other patients.

\section{Discussion}

There were several differences between the primary tumors and the corresponding metastases, especially with regard to the expression of HER2 and HER3. HER2 was upregulated in the metastases of 5 out of the 8 patients with HER2-negative primary tumors. There was positive co-expression of EGFR and HER 2 in only one of the primary tumors, while there was positive co-expression of EGFR and HER2 in 4 of the metastases. Thus, there was a tendency for both upregulation 
of HER2 and increased positive co-expression of EGFR and HER2 in the lymph node metastases in comparison to the primary tumors. Furthermore, there appeared to be a marked downregulation of HER3 since 7 metastases had downregulated HER3, from weak or strong expression in the primary tumors to negative in the corresponding metastases.

Thus, it appears that the tumor cells forming the metastases may be a subtype, most likely with a more aggressive phenotype and/or genotype than the cells in the primary tumors. However, there was no downregulation of EGFR, indicating that the metastases originating from EGFR-positive primary tumors may be dependent on continued EGFR expression. It has been indicated that blocking EGFR reduces the invasive potential of prostate cancer cells $(22,23)$.

The EGFR expression frequency in primary prostate cancer is, according to the few available previously published studies, in the range of $40-45 \%$ and is higher in hormone refractory (castration resistant) than in hormone sensitive prostate cancers $(2,24-26)$. In the present study, 5 out of the 12 patients had EGFR-positive primary tumors.

The HER2 expression frequency in hormone refractory (castration resistant) prostate cancer has been reported in in wide range $20-70 \%(2,27-30)$. Thus, there are studies on both high and low frequencies of HER2 expression in the primary tumors and one study reported almost no HER2 expression (31). However, HER2 has been reported to be expressed at high frequencies in metastases from prostate cancer and has, in one study, been found in up to $90 \%$ of the analyzed cases (32). In the present study, 8 out of 12 metastases were HER2-positive. This indicates upregulation of HER2 in metastases. HER2-positive prostate cancer cells have also been detected in the peripheral blood of prostate cancer patients (33). By contrast, it has been reported that HER2 was expressed in metastases at a similar level as in the corresponding primary prostate tumors (34) and one study reported almost no HER2 expression in lymph node metastases from prostate cancer (31).

The reported variations between different studies on HER2 expression are probably due to different patient inclusion and receptor scoring criteria and there are also differences in immunohistochemical retrieval techniques between the laboratories, as can be observed in the original articles. It is important to agree on common histological processing techniques and standardized scoring criteria and the HercepTest applied for breast cancer (21) is a good example. The possibility that etiological differences play a role in the variation of reported HER2 expression levels cannot be excluded.

The HER3 expression frequency has been reported to be $21 \%$ in primary prostate cancers (27) and HER3 appears to be expressed in metastases $(2,34)$. However, the indicated downregulation of HER3 in prostate metastases in the present study has not previously been reported. A secreted isoform of HER3, MDA-BF-1, has been observed in metastatic prostate cancer (35).

There is a controversy since molecular biology studies report HER 3 to be a cell membrane-associated receptor expressing a transmembrane region. This is in contrast to a number of histopathological findings, with most of the HER3 staining in the cytoplasm. It cannot be excluded that HER3 is, to a large extent, associated with intracellular membranes and/or that precursors of HER3 in the cytoplasm are stained.
Furthermore, HER3 may be in the outer cell membrane for only a short time due to a possible rapid turnover.

The expression of EGFR and HER2 in normal tissues was characterized a number of years ago. EGFR is expressed in the skin, liver, digestive tract and reproductive organs (36-38). The distributions of EGFR in various tissues can also be found at the human protein atlas (http://www.proteinatlas.org/). The HER2 expression in normal tissues in adults is generally low (39-41) (see also the human protein atlas: http://www. proteinatlas.org/).

The previously published receptor determinations, together with our findings, indicate that prostate cancers have the potential to express EGFR and HER2 in primary tumors and metastases. It is noteworthy that four patients (numbers 2, 4, 7 and 12) out of the 12 studied may be candidates for combined therapy, targeting both EGFR and HER2 in the metastases, at least if the analyzed lymph node metastases are representative of any other metastases that these four patients may suffer from. The targeting process should then deliver radionuclides or toxins of therapeutic use. This may be necessary since HER2-targeted therapy of hormone refractory (castration resistant) prostate cancers with antibodies without toxic agents, 'naked antibodies', has recently been studied, thus far without positive results $(42,43)$. Furthermore, the use of tyrosine kinase inhibitors blocking both HER2 and EGFR in hormone refractory (castration resistant) prostate cancers has also not shown a good response (44), although there is hope for future improvements $(45,46)$. Delivery of toxic agents via the receptors is most likely a better choice.

Thus, targeted therapy delivering radionuclides or toxins is an alternative in cases with significant levels of receptors, but with the tumor cells resistant to chemotherapy, tyrosine kinase inhibitors and the action of 'naked antibodies'. However, the expression intensity per tumor cell of EGFR and HER2 in prostate cancer metastases may be low and/or heterogeneous (3). This further indicates that there may be a need for 'multiple targeting', e.g., a cocktail with binders to both EGFR and HER2. The possibility to target more than one receptor at the time should be considered since co-expression of receptors may also be associated with high-grade malignancy $(6,7)$ and targeting against EGFR and HER2 may also increase the targeting specificity. More research is needed regarding this.

We are especially interested in targeted radionuclide therapy since that relies on several years of clinical experience to kill tumor cells and severe resistance has thus far not been associated with radiation therapy. Targeted radionuclide therapy using radiolabeled somatostatin analogs $\left({ }^{177} \mathrm{Lu}\right.$-Octreotate) for treatment of neuroendocrine tumors and radiolabeled anti-CD20 antibodies $\left({ }^{90}\right.$ Y-Zevalin) for treatment of chemotherapy-resistant lymphomas are accepted modalities $(1,2)$. The promising therapeutic results in these cases suggest that targeted radionuclide therapy may also be successful in the treatment of prostate cancers and that more patients may be treated with a curative instead of palliative intention.

The design of suitable receptor-binding agents with high binding to prostate cancer cells and low uptake in critical normal tissues is a challenge. However, there is potential for development since new knowledge is continuously emerging about biodistribution, pharmacokinetics and the cellular processing of different types of targeting agents and 
the research on molecular design of new targeting agents is rapidly expanding (47). The development of peptides and small proteins, such as affibody molecules (48), is one strategy and the area of antibody engineering is rapidly developing. Various forms of antibody fragments, including minimal recognizing units, single chain fragments ( $\mathrm{scFvs}$ ) and dimeric scFvs, are drawing increasing interest $(49,50)$. Bifunctional molecules, with capacity to bind two different receptors at the same time are, as indicated above, a possible approach for therapy. Liposomes containing toxic substances and conjugated with targeting agents may be of use for the killing of disseminated tumor cells in the systemic circulation (51).

To summarize, the indicated increase in HER2 expression and co-expression of EGFR and HER2 in the metastases may be promising for the use of agents that deliver therapeutically useful radionuclides or toxins in at least a subpopulation of prostate cancer patients. HER3 may be of less use, based on the results of the present limited study. The results are encouraging for studies involving more patients.

\section{Acknowledgements}

This study was funded by the Swedish Cancer Society (no. 11 0565; J. Carlsson) and grants from National Natural Science Foundation of China (no. 81071823; Q. Wei) and from the Young Investigator Fund from Health Bureau of Zhejiang China (no. 2008QN020; Q. Wei).

\section{References}

1. Sharkey RM and Goldenberg DM: Cancer radioimmunotherapy. Immunotherapy 3: 349-370, 2011.

2. Carlsson J: Potential for clinical radionuclide-based imaging and therapy of common cancers expressing EGFR-family receptors. Tumour Biol 33: 653-659, 2012.

3. Carlsson J: EGFR-family expression and implications for targeted radionuclide therapy. In: Targeted Radionuclide Tumor Therapy, Biological Aspects. Stigbrand T, Adams G and Carlsson J (eds) Springer Verlag, pp 25-58, 2008.

4. Houssami N, Macaskill P, Balleine RL, Bilous M and Pegram MD HER2 discordance between primary breast cancer and its paired metastasis: tumor biology or test artefact? Insights through metaanalysis. Breast Cancer Res Treat 129: 659-674, 2011.

5. Pecorino L (ed): Molecular Biology of Cancer: Mechanisms, Targets, and Therapeutics. Oxford University Press, Oxford, 2005.

6. Citri A and Yarden Y: EGF-ERBB signalling: towards the systems level. Nat Rev Mol Cell Biol 7: 505-516, 2006.

7. Bublil EM and Yarden Y: The EGF receptor family: spearheading a merger of signaling and therapeutics. Curr Opin Cell Biol 19: 124-134, 2007.

8. Morgillo F, Bareschino MA, Bianco R, Tortora G and Ciardiello F: Primary and acquired resistance to anti-EGFR targeted drugs in cancer therapy. Differentiation 75: 788-799, 2007.

9. Lièvre A, Bachet JB, Boige V, Cayre A, Le Corre D, Buc E, Ychou M, Bouché O, Landi B, Louvet C, et al: KRAS mutations as an independent prognostic factor in patients with advanced colorectal cancer treated with cetuximab. J Clin Oncol 26: 374-379, 2008

10. Di Nicolantonio F, Martini M, Molinari F, Sartore-Bianchi A, Arena S, Saletti P, De Dosso S, Mazzucchelli L, Frattini M, Siena $\mathrm{S}$ and Bardelli A: Wild-type BRAF is required for response to panitumumab or cetuximab in metastatic colorectal cancer. $\mathrm{J}$ Clin Oncol 26: 5705-5712, 2008.

11. Nahta R and Esteva FJ: Trastuzumab: triumphs and tribulations Oncogene 26: 3637-3643, 2007.

12. Berns K, Horlings HM, Hennessy BT, Madiredjo M, Hijmans EM, Beelen K, Linn SC, Gonzalez-Angulo AM, Stemke-Hale K, Hauptmann M, et al: A functional genetic approach identifies the PI3K pathway as a major determinant of trastuzumab resistance in breast cancer. Cancer Cell 12: 395-402, 2007.
13. Jathal MK, Chen L, Mudryj M and Ghosh PM: Targeting ErbB3: the New RTK(id) on the Prostate Cancer Block. Immunol Endocr Metab Agents Med Chem 11: 131-149, 2011.

14. Wei Q, Sheng L, Shui Y, Hu Q, Nordgren H and Carlsson J: EGFR, HER2, and HER3 expression in laryngeal primary tumors and corresponding metastases. Ann Surg Oncol 15: 1193-1201, 2008.

15. Wei Q, Chen L, Sheng L, Nordgren H, Wester K and Carlsson J: EGFR, HER2 and HER3 expression in esophageal primary tumours and corresponding metastases. Int J Oncol 31: 493-499, 2007.

16. Ekberg T, Nestor M, Engström M, Nordgren H, Wester K, Carlsson J and Anniko M: Expression of EGFR, HER2, HER3, and HER4 in metastatic squamous cell carcinomas of the oral cavity and base of tongue. Int J Oncol 26: 1177-1185, 2005.

17. Wei Q, Shui Y, Zheng S, Wester K, Nordgren H, Nygren P, Glimelius B and Carlsson J: EGFR, HER2 and HER3 expression in primary colorectal carcinomas and corresponding metastases: Implications for targeted radionuclide therapy. Oncol Rep 25: 3-11, 2011.

18. Gleason DF; The Veteran's Administration Cooperative Urologic Research Group: Histologic grading and clinical staging of prostatic carcinoma. In: Urologic Pathology: The Prostate. Tannenbaum M (ed). Lea and Febiger, Philadelphia, pp171-198, 1977.

19. Gleason DF: Histology grading of prostate cancer: a perspective. Hum Path 23: 273-279, 1992.

20. Held-Warmkessel J (ed): Contemporary Issues in Prostate Cancer: A Nursing Perspective. Jones \& Bartlett Learning, pp107-125, 2006.

21. Bilous M, Dowsett M, Hanna W, Isola J, Lebeau A, Moreno A, Penault-Llorca F, Rüschoff J, Tomasic G and van de Vijver M: Current perspectives on HER2 testing: a review of national testing guidelines. Mod Pathol 16: 173-182, 2003.

22. Kim SJ, Uehara H, Karashima T, Shepherd DL, Killion JJ and Fidler IJ: Blockade of epidermal growth factor receptor signaling in tumor cells and tumor-associated endothelial cells for therapy of androgen-independent human prostate cancer growing in the bone of nude mice. Clin Cancer Res 9: 1200-1210, 2003.

23. Festuccia C, Angelucci A, Gravina GL, Biordi L, Millimaggi D, Muzi P, Vicentini C and Bologna M: Epidermal growth factor modulates prostate cancer cell invasiveness regulating urokinasetype plasminogen activator activity. EGF-receptor inhibition may prevent tumor cell dissemination. Thromb Haemost 93: 964-975, 2005.

24. Scher HI, Sarkis A, Reuter V, Cohen D, Netto G, Petrylak D, Lianes P, Fuks Z, Mendelsohn J and Cordon-Cardo C: Changing pattern of expression of the epidermal growth factor receptor and transforming growth factor alpha in the progression of prostatic neoplasms. Clin Cancer Res 1: 545-550, 1995.

25. Shah RB, Ghosh D and Elder JT: Epidermal growth factor receptor (ErbB1) expression in prostate cancer progression: correlation with androgen independence. Prostate 66: 1437-1444, 2006.

26. Festuccia C, Gravina GL, Millimaggi D, Muzi P, Speca S, Ricevuto E, Vicentini C and Bologna M: Uncoupling of the epidermal growth factor receptor from downstream signal transduction molecules guides the acquired resistance to gefitinib in prostate cancer cells. Oncol Rep 18: 503-511, 2007.

27. Hernes E, Fossá SD, Berner A, Otnes B and Nesland JM: Expression of the epidermal growth factor receptor family in prostate carcinoma before and during androgen-independence. Br J Cancer 90: 449-454, 2004.

28. Bartlett JM, Brawley D, Grigor K, Munro AF, Dunne B and Edwards J: Type I receptor tyrosine kinases are associated with hormone escape in prostate cancer. J Pathol 205: 522-529, 2005.

29. Morote J, de Torres I, Caceres C, Vallejo C, Schwartz S Jr and Reventos J: Prognostic value of immunohistochemical expression of the c-erbB-2 oncoprotein in metastasic prostate cancer. Int J Cancer 84: 421-425, 1999.

30. Reese DM, Small EJ, Magrane G, Waldman FM, Chew K and Sudilovsky D: HER2 protein expression and gene amplification in androgen-independent prostate cancer. Am J Clin Pathol 116: 234-239, 2001.

31. Liu HL, Gandour-Edwards R, Lara PN Jr, de Vere White R and LaSalle JM: Detection of low level HER-2/neu gene amplification in prostate cancer by fluorescence in situ hybridization. Cancer J 7: 395-403, 2001.

32. Carles J, Lloreta J, Salido M, Font A, Suarez M, Baena V, Nogue M, Domenech M and Fabregat X: Her-2/neu expression in prostate cancer: a dynamic process? Clin Cancer Res 10: 4742-4745, 2004. 
33. Ady N, Morat L, Fizazi K, Soria JC, Mathieu MC, Prapotnich D, Sabatier L and Chauveinc L: Detection of HER-2/neu-positive circulating epithelial cells in prostate cancer patients. Br J Cancer 90: 443-448, 2004.

34. Myers RB, Srivastava S, Oelschlager DK and Grizzle WE: Expression of p160erbB-3 and p185erbB-2 in prostatic intraepithelial neoplasia and prostatic adenocarcinoma. J Natl Cancer Inst 86: 1140-1145, 1994.

35. Vakar-Lopez F, Cheng CJ, Kim J, Shi GG, Troncoso P, Tu SM, Yu-Lee LY and Lin SH: Up-regulation of MDA-BF-1, a secreted isoform of ErbB3, in metastatic prostate cancer cells and activated osteoblasts in bone marrow. J Pathol 203: 688-695, 2004.

36. Gusterson B, Cowley G, Smith JA and Ozanne B: Cellular localisation of human epidermal growth factor receptor. Cell Biol Int Rep 8: 649-658, 1984.

37. Damjanov I, Mildner B and Knowles BB: Immunohistochemical localization of the epidermal growth factor receptor in norma human tissues. Lab Invest 55: 588-592, 1986.

38. Dittadi R, Gion M, Pagan V, Brazzale A, Del Maschio O, Bargossi A, Busetto A and Bruscagnin G: Epidermal growth factor receptor in lung malignancies. Comparison between cancer and normal tissue. Br J Cancer 64: 741-744, 1991.

39. Natali PG, Nicotra MR, Bigotti A, Venturo I, Slamon DJ, Fendly BM and Ullrich A: Expression of the p185 encoded by HER 2 oncogene in normal and transformed human tissues. Int J Cancer 45: 457-461, 1990.

40. Press MF, Cordon-Cardo C and Slamon DJ: Expression of the HER-2/neu proto-oncogene in normal human adult and fetal tissues. Oncogene 5: 953-962, 1990.

41. Gutierrez C and Schiff R: HER 2: biology, detection, and clinical implications. Arch Pathol Lab Med 135: 55-62, 2011.

42. Agus DB, Sweeney CJ, Morris MJ, Mendelson DS, McNeel DG, Ahmann FR, Wang J, Derynck MK, Ng K, Lyons B, Allison DE, Kattan MW and Scher HI: Efficacy and safety of single-agent pertuzumab (rhuMAb 2C4), a human epidermal growth factor receptor dimerization inhibitor, in castration-resistant prostate cancer after progression from taxane-based therapy. J Clin Oncol 25: 675-681,2007.
43. de Bono JS, Bellmunt J, Attard G, Droz JP, Miller K, Flechon A, Sternberg C, Parker C, Zugmaier G, Hersberger-Gimenez V, et al: Open-label phase II study evaluating the efficacy and safety of two doses of pertuzumab in castrate chemotherapy-naive patients with hormone-refractory prostate cancer. J Clin Oncol 25: 257-262, 2007.

44. Solit DB and Rosen N: Targeting HER 2 in prostate cancer: where to next? J Clin Oncol 25: 241-243, 2007.

45. Gallick GE, Corn PG, Zurita AJ and Lin SH: Small-molecule protein tyrosine kinase inhibitors for the treatment of metastatic prostate cancer. Future Med Chem 4: 107-119, 2012.

46. Chen L, Mooso BA, Jathal MK, Madhav A, Johnson SD, van Spyk E, Mikhailova M, Zierenberg-Ripoll A, Xue L, Vinall RL, et al: Dual EGFR/HER2 inhibition sensitizes prostate cancer cells to androgen withdrawal by suppressing ErbB3. Clin Cancer Res 17: 6218-6228, 2011

47. Stigbrand T, Carlsson J and Adams GP: Developmental trends in targeted radionuclide therapy - biological aspects. In: Targeted Radionuclide Tumor Therapy, Biological Aspects. Stigbrand T, Adams G and Carlsson J (eds). Springer Verlag, pp 387-397, 2008.

48. Frejd YF: Novel alternative scaffolds and their potential use for tumor targeted radionuclide therapy. In: Targeted Radionuclide Tumor Therapy, Biological Aspects. Stigbrand T, Adams G and Carlsson J (eds). Springer Verlag, pp 89-116, 2008.

49. Govindan SV and Goldenberg DM: New antibody conjugates in cancer therapy. Scientific World Journal 10: 2070-2089, 2010.

50. Adams GP and Weiner LM: Monoclonal antibody therapy of cancer. Nat Biotechnol 23: 1147-1157, 2005.

51. Gedda L, Fondell A, Lundqvist H, Park JW and Edwards K: Experimental radionuclide therapy of HER2-expressing xenografts using two-step targeting nuclisome particles. J Nucl Med 53: 480-487, 2012. 